TRANSACTIONS OF THE

AMERICAN MATHEMATICAL SOCIETY

Volume 00, Number 0, Pages 000-000

S $0002-9947(\mathrm{XX}) 0000-0$

\title{
ENERGY DISTRIBUTION OF A GÖDEL-TYPE SPACE-TIME
}

\author{
RAGAB M. GAD
}

\begin{abstract}
We calculate the energy and momentum distributions associated with a Gödel-type space-time, using the well-known energy-momentum complexes of Landau-Lifshitz and Møller. We show that the definitions of LandauLifshitz and Møller do not furnish a consistent result.
\end{abstract}

\section{INTRODUCTION}

One of the most active areas of research in the general theory of relativity is the energy-momentum complex. There are many attempts to evaluate the energy distribution in a general relativistic system beginning with the Einstein's energy-momentum complex and followed by many prescriptions, for instance, Landau-Lifshitz (LL), Papapetrou and Weinberg (See in [1, 2] and references therein). Most of these prescriptions only giving meaningful results if the calculations are performed in "Cartesian coordinates". Møller [3] constructed an expression which enables one to evaluate energy in any coordinate system.

Several examples of particular space-times (the Kerr-Newman, the EinsteinRosen and the Bonnor-Vaidya) have been investigated and different energy momentum complexes are known to give the same energy distribution for a given space-time [4] - 10]. Vagenas [11 has proved that in $(2+1) d$ all complexes give the same results. Virbhadra and Parikh [12, 13] studied the energy distribution associated with a stringy charged black-hole in Einstein's prescription. The same result has been obtained by Xulu [14] using Tolman's energy-momentum complex that is in fact the Einstein energy-momentum complex expressed by Tolman in a different form (see [4] for details.)

Virbhadra [1] noted that the definitions of LL, Papaperrou and Weinberg give the same result as in the Einstein prescription if the calculations are performed in Kerr-Schild Cartesian coordinates. However, these complexes disagree with Einstein definition if computations are done in "Schwarzschild Cartesian coordinates ${ }^{1}$."

Recently, Xulu [15] obtained the energy distribution for the most general non-static spherically symmetric metric using Møller's definition. He

\footnotetext{
${ }^{1}$ Schwarzschild metric in "Schwarzschild Cartesian coordinates" is obtained by transforming this metric (in usual Schwarzschild coordinates $\{t, r, \theta, \phi\}$ ) to $\{t, x, y, z\}$ using $x=r \sin \theta \cos \phi, x=$ $r \sin \theta \sin \phi, z=r \cos \theta$.
} 
found different results in general from those obtained using the Einstein energy momentum complex; these results agree for the Schwarzschild, Vaidya and Janis-Newman-Winicour space-times, but disagree for the ReissnerNordström space-time.

Sharif [16] evaluated the energy and momentum density components for Gödel-type metric by using prescriptions of Einstein and Papapetrou. He found that the two prescriptions differ in general for this space-time. Dabrowski [17. has calculated the energy momentum and angular momentum, using Einstein and Bergmann definitions, for both acausal and causal Gödel model.

In this paper we will evaluate the energy and momentum densities for a Gödel-type space-time using the energy-momentum complexes of LandauLifshitz and Møller. We obtain them if the space-time under consideration is homogeneous. We are also interested to check whether or not the Cooperstock hypothesis [18. ( which states that the energy and momentum in a curved space-time are confined to the regions of non-vanishing energymomentum tensor $T_{i}^{k}$ of the matter and all non-gravitational fields) holds for this case.

Through this paper we use $G=1$ and $c=1$ units and follow the convention that Latin indices take value from 0 to 3 and Greek indices take value from 1 to 3 .

\section{GÖDEL-TYPE SPACE-TIME}

An exact solution of Einstein's field equations with cosmological constant for incoherent matter with rotation has been found by Gödel in 1949 19. This solution, called Gödel-type manifold, described by the line element [20]

$$
d s^{2}=[d t+H(r) d \phi]^{2}-D^{2}(r) d \phi^{2}-d r^{2}-d z^{2},
$$

where $r, \phi$ and $z$ are cylindrical coordinates.

The non-zero components of the energy-momentum tensor for the Gödeltype metric (2.1) are

$$
\begin{aligned}
& T_{0}^{0}=-\frac{1}{8 \pi}\left(\frac{D^{\prime \prime}}{D}-3\left(\frac{H^{\prime}}{2 D}\right)^{2}-\frac{H}{2 D}\left(\frac{H^{\prime}}{D}\right)^{\prime}\right), \\
& T_{2}^{0}=-\frac{1}{8 \pi}\left[\frac{H D^{\prime \prime}}{D}-\frac{D}{2}\left(\frac{H^{\prime}}{D}\right)^{\prime}-\frac{3 H}{4}\left(\frac{H^{\prime}}{D}\right)^{2}-\frac{H^{2}}{2 D}\left(\frac{H^{\prime}}{D}\right)^{\prime}-\frac{H^{3}}{D^{2}}\left(\frac{H^{\prime}}{2 H}\right)^{2}\right], \\
& T_{1}^{1}=-\frac{1}{8 \pi}\left(\frac{H^{\prime}}{2 D}\right)^{2} \\
& T_{2}^{2}=-\frac{1}{8 \pi}\left[\frac{H}{2 D}\left(\frac{H^{\prime}}{D}\right)^{\prime}++\frac{H^{2}}{D^{2}}\left(\frac{H^{\prime}}{2 H}\right)^{2}\right] \\
& T_{3}^{3}=-\frac{1}{8 \pi}\left[-\left(\frac{H^{\prime}}{2 D}\right)^{2}+\frac{D^{\prime \prime}}{D}\right] .
\end{aligned}
$$

The Gödel cosmological solution has a well-recognized importance and has to a large extent motivated the investigations on rotating cosmological spacetime within the frame work of general relativity. Particularly, the search for 
rotating Gödel-type space-times has received a good deal attention in recent years, and the literature on these geometries is fairly large today (See Refs. 21 - 29 and references therein).

The homogeneity of this space-time was considered for the first time in 1980 by Raychandhuri and Thakurta 30. The necessary and sufficient conditions for a Gödel-type manifold (2.1) to be space-time homogeneous were proved in 31. These space-times are characterized by metrics of the form (2.1) which, in addition, satisfy the following restrictions

$$
\frac{D^{\prime \prime}}{D}=m^{2}=\text { const. and } \frac{H^{\prime}}{D}=\text { const. }=2 \Omega,
$$

where prime denotes the differentiation w.r.t. $r$.

It is well known that the energy-momentum complexes give meaningful result if they are evaluated in Cartesian coordinates. According to the following transformations

$$
x=r \cos \phi, \quad y=r \sin \phi, \quad z=z,
$$

and

$$
r=\sqrt{x^{2}+y^{2}}
$$

the line element (2.1) may be transformed to quasi-Cartesian coordinates:

$$
\begin{gathered}
d s^{2}=d t^{2}+2 H(r)\left(\frac{x}{r^{2}} d y-\frac{y}{r^{2}} d x\right) d t-\frac{\left(D^{2}(r)-H^{2}(r)\right)}{r^{4}}(x d y-y d x)^{2} \\
-\frac{1}{r^{2}}(x d x+y d y)^{2}-d z^{2} .
\end{gathered}
$$

The determinant of the metric tensor is

$$
g=-\frac{D^{2}}{r^{2}}
$$

The components of the contravariant metric tensor are

$$
\begin{aligned}
g^{00} & =\frac{D^{2}-H^{2}}{D^{2}}, \\
g^{01} & =-\frac{y H}{D^{2}}, \\
g^{02} & =\frac{x H}{D^{2}}, \\
g^{11} & =-\frac{x^{2} D^{2}+y^{2} r^{2}}{r^{2} D^{2}}, \\
g^{12} & =-\frac{x y\left(D^{2}-r^{2}\right)}{r^{2} D^{2}}, \\
g^{22} & =-\frac{y^{2} D^{2}+x^{2} r^{2}}{r^{2} D^{2}}, \\
g^{33} & =-1 .
\end{aligned}
$$




\section{Energy AND MOMEntum in LANDAU-Lifshitz's Prescription}

The energy and momentum densities in the sense of Landau-Lifshitz 40] are given by

$$
P^{m}=\frac{1}{16 \pi} S_{, j k}^{m j 0 k}
$$

where

$$
S^{m j n k}=-g\left(g^{m n} g^{j k}-g^{m k} g^{j n}\right),
$$

and has symmetries of the Riemann tensor.

$P^{0}$ is the energy density and $P^{\alpha}$ are the momentum density components.

In order to calculate the energy and momentum densities for Gödel-type expressed by the line element (2.1) we need the following non-zero components of $S^{m j n k}$

$$
\begin{aligned}
& S^{0101}=\frac{x^{2}}{r^{4}}\left(H^{2}-D^{2}\right)-\frac{y^{2}}{r^{2}}, \\
& S^{0102}=\frac{x y}{r^{4}}\left(H^{2}-D^{2}\right)+\frac{x y}{r^{2}}, \\
& S^{0202}=\frac{y^{2}}{r^{4}}\left(H^{2}-D^{2}\right)-\frac{x^{2}}{r^{2}}, \\
& S^{1201}=\frac{x H}{r^{2}}, \\
& S^{1202}=\frac{y H}{r^{2}} .
\end{aligned}
$$

Substituting (3.3) in (3.1), one gets the energy and momentum densities in Landau-Lifshitz as

$P^{0}=\frac{1}{8 \pi r^{4}}\left(\left(H^{2}-D^{2}\right)-3 r\left(H H^{\prime}-D D^{\prime}\right)+r^{2}\left(H^{\prime 2}-D^{\prime 2}+H H^{\prime \prime}-D D^{\prime \prime}\right)\right)$,

$$
\begin{aligned}
P^{x} & =\frac{y}{16 \pi r^{3}}\left(r H^{\prime \prime}-H^{\prime}\right), \\
P^{y} & =\frac{x}{16 \pi r^{3}}\left(H^{\prime}-r H^{\prime \prime}\right), \\
P^{z} & =0 .
\end{aligned}
$$

The line element (2.1) with $H=e^{a r}, D=\frac{e^{a r}}{\sqrt{2}}, a=-m$, and $m^{2}=2 \Omega^{2}$ is homogenous in space and time (hereafter called ST-homogeneous). Hence, 
from equation (2.2), the non-vanishing components of $T_{b}^{a}$ become.

$$
\begin{aligned}
& T_{0}^{0}=\frac{\Omega^{2}}{8 \pi}, \\
& T_{2}^{0}=\frac{\Omega^{2}}{4 \pi}, \\
& T_{1}^{1}=-\frac{\Omega^{2}}{8 \pi}, \\
& T_{2}^{2}=-\frac{\Omega^{2}}{8 \pi}, \\
& T_{3}^{3}=-\frac{\Omega^{2}}{8 \pi} .
\end{aligned}
$$

From equations (3.4) the energy and momentum densities for ST-homogeneous become

$$
\begin{aligned}
P^{0} & =\frac{e^{2 a r}}{16 \pi r^{4}}(r-1)(2 r-1), \\
P^{x} & =\frac{a y e^{a r}}{16 \pi r^{3}}(a r-1), \\
P^{y} & =\frac{a x e^{a r}}{16 \pi r^{3}}(1-a r) .
\end{aligned}
$$

It is worthy to investigate the Cooperstock hypothesis for the ST-homogeneous. We see that when $r=0$, the components of energy momentum tensor (3.5) have finite values, while from the equations (3.6) the energy and momentum components tend to infinity. Therefore the results obviously not uphold the Cooperstock hypothesis.

\section{Energy Distribution in Møller's Prescription}

Møller's energy-momentum complex [3] is given by

$$
\Theta_{i}^{k}=\frac{1}{8 \pi} \chi_{i, l}^{k l}
$$

where the antisymmetric superpotential $\chi_{i}^{k l}$ is

$$
\chi_{i}^{k l}=-\chi_{i}^{l k}=\sqrt{-g}\left(\frac{\partial g_{i n}}{\partial x^{m}}-\frac{\partial g_{i m}}{\partial x^{n}}\right) g^{k m} g^{n l},
$$

$\Theta_{0}^{0}$ is the energy density and $\Theta_{\alpha}^{0}$ are the momentum density components. Also, the energy-momentum complex $\Theta_{i}^{k}$ satisfies the local conservation laws:

$$
\frac{\partial \Theta_{i}^{k}}{\partial x^{k}}=0
$$


The only non-vanishing components of $\chi_{i}^{k l}$ are

$$
\begin{aligned}
\chi_{0}^{01} & =\frac{H H^{\prime}}{D} \\
\chi_{2}^{01} & =\frac{1}{D}\left(H^{\prime}\left(H^{2}+D^{2}\right)-2 D D^{\prime} H\right) .
\end{aligned}
$$

Using the above components in (4.1), we obtain the energy and momentum densities in the form

$$
\begin{aligned}
& \Theta_{0}^{0}=\frac{1}{8 \pi D^{2}}\left(D H H^{\prime \prime}+D H^{\prime 2}-H H^{\prime} D^{\prime}\right), \\
& \Theta_{2}^{0}=\frac{1}{8 \pi D^{2}}\left[\left(H^{2}+D^{2}\right)\left(D H^{\prime \prime}-H^{\prime} D^{\prime}\right)+2 H D\left(H^{\prime 2}-D D^{\prime \prime}\right],\right. \\
& \Theta_{1}^{0}=\Theta_{3}^{0}=0 .
\end{aligned}
$$

For the ST-homogeneous Gödel-type space-time, the energy and momentum densities become

$$
\begin{aligned}
& \Theta_{0}^{0}=\frac{m^{2} e^{-m r}}{4 \sqrt{2} \pi}, \\
& \Theta_{2}^{0}=\frac{m^{2} e^{-2 m r}}{4 \sqrt{2} \pi}, \\
& \Theta_{1}^{0}=\Theta_{3}^{0}=0 .
\end{aligned}
$$

We note that from equation (4.6), if $r$ tends to infinity the energy and momentum components tend to zero, but from equation (3.5) the energy momentum tensor componebts, $T_{b}^{a}$, have finite values. Therefore the Møller's definition not upholds the Cooperstock hypothesis for the ST-homogeneous.

\section{DisCUSSION}

Ever since the first investigations of cylindrically symmetric space-times by Levi-Civita 33] and, latter by Lewis [34, these space-times have been studied extensively for their mathematical and physical properties. These have recently been studied particularly in context of black hole 35], gravitational waves and cosmic strings [36. Some examples of well known cylindrically symmetric astrophysical and cosmological solutions discussed in the literature include Einstein-Maxwell fields [37, magnetic strings [38, static gravitational fields [39] and a large number of cosmic string solutions.

Using different definitions of energy-momentum complex, several authors studied the energy distribution for a given space-time. Most of them restricted their intention to the static and non-static spherically symmetric space-times.

In the present work we investigated the energy and momentum associated with the space-time in cylindrical coordinates using Landau-Lifshitz and Møller definitions. We obtained the energy and momentum density components of the Gödel-type space-time as well as the homogeneous Gödel-type space-time in these two prescriptions. We found that the two definitions of 
energy-momentum complexes do not provide the same result for these type of metrics.

The homogenous Gödel-type space-time provide one more example where the energy and momentum localization together with the Cooperstock hypothesis are far from resolved.

\section{REFERENCES}

[1] K. S. Virbhadra, Phys. Rev. D 60, 104041 (1999).

[2] J. M. Aguirregabiria, A. Chamorro and K. S. Virbhadra, Gen. Relativ. Gravit. 28, 1393 (1996).

[3] C. Møller, Annals of Physics (NY), 4, 347 (1958).

[4] K. S. Virbhadra, Phys. Rev. D, 41, 1086 (1990).

[5] K. S. Virbhadra, Phys. Rev. D, 42, 1066 (1990).

[6] K. S. Virbhadra, Phys. Rev. D, 42, 2919 (1990).

[7] A. Chamorro and K. S. Virbhadra, Pramana-Journal of Physics, 45, 181 (1995).

[8] Tod K. P. (1983). Proc. R. Soc. London A 388, 467 (1983).

[9] N. Rosen and K. S. Virbhadra Gen. Relativ. Gravit., 25, 429 (1993).

[10] K. S. Virbhadra, Pramana-Journal of Physics, 45, 215 (1995).

[11] E. C. Vagenas, hep-th/0307162

[12] K. S. Virbhadra and J. C. Parikh, Phys. Lett. B, 317, 312 (1993).

[13] K. S. Virbhadra and J. C. Parikh, Phys. Lett. B, 331, 302 (1994).

[14] S. S. Xulu, International Journal of Theoretical Physics, 37, 1773 (1998).

[15] S. S. Xulu gr-qc/0010068

[16] M. Shraif, gr-qc/0310018

[17] M. P. Dabrowski, gr-qc/03090664.

[18] F. I. cooperstock, Mod. Phys. Lett. A14 1531 (1999).

[19] K. Gödel, Rev. Mod. Phys. 21, 447 (1949).

[20] M. J. Reboucas and J. Tiomno, Phys. Rev. B 28, 1251 (1983).

[21] M. M. Som and A. K. Raychaudhuri, Proc. R. Soc. London, Ser. A 304, 81 (1968).

[22] F. Bampi and C. Zordan, Gen. Relativ. Gravit. 9, 393 (1978).

[23] M. J. Reboucas, Phys. Lett. 70, 161 (1979).

[24] J. Pfarr, Gen. Relativ. Gravit. 13, 1073 (1981).

[25] S. K. Chakraborty, Gen. Relativ. Gravit. 12, 925 (1980).

[26] M. J. Reboucas, J. E. Aman, and F. F. Taxeira, J. Math. Phys. 27, 1370 (1986).

[27] M. J. Reboucas, and F. F. Taxeira, Phys. Rev. D 34, 2985 (1986).

[28] F. M. Paiva, M. J. Reboucas, and F. F. Taxeira, Phys. Lett. A 126, 168 (1987).

[29] F. M. Saibatalov, Gen. Relativ. Gravit. 17, 697 (1995).

[30] A. K. Raychaudhuri and S. N. G. Thakurta, Phys. Rev. D 22, 802 (1980).

[31] A. F. F. Teixeira, M.J. Reboucas, and J. E. Aman, Phys. Rev D 32, 3309 (1985).

[32] R. C. Tolman, "Relativity, Thermodynamics and Cosmology, (Oxford University Press, Oxford), (1934) p. 227.

[33] T. Levi-Civita, Rend. Acc. Lincei 26, 307 (1917).

[34] T. Lewis, Proc. R. Soc. London A136, 176 (1932).

[35] J. P. S. Lemos, Phys. Lett. B 353, 46 (1995), and Phys. Rev. D 57, 4600 (1998).

[36] S. A. Hayward, Class. Quantum Grav., 17, 1749 (2000).

[37] C. Liang, Gen. Relativ. Gravit., 27, 669 (191995).

[38] O. J. C. Dias and J. P. S., Class. Quantum Grav., 19, 2265 (2002).

[39] J. Colding, N. K. Nielsen and Y. Vorbin, Phys. Rev. D 56, 3371 (1997).

[40] L. D. Landau and E. M. Lifshitz, The Classical Theory of Fields (Pergamon, Oxford, 1985) p. 280. 
Mathematics Department, Faculty of Science, Minia University, El-Minia, Egypt

E-mail address: ragab2gad@hotmail.com 